\title{
Desarrollo participativo y la apropiación de la agencia: Una exploración sociológica
}

\author{
- Salvador Orlando Alfaro \\ Departamento de Sociologia y Estudios Sociales \\ Universidad de Regina (Canadá)
}

La comprensión del desarrollo como discurso, el cual como el orienralismo, lunciona como un disposilivo ideológico, ha sido lundamental en el progreso que ha experimentado la narrativa del desarrollo (Long y long 1992). Esto ha contribuido a centrar el análisis en el vínculo que existe entre las diferentes formas de conocimiento y la manera en que se enlientan en la implementación de los proyecios de desarrollo, lo que su ves ha estimulado una evaluación más rigurosa sobre las dilerentes pereepciones y elaboraciones acerea del denominado "conocimiento local". Asimismo, estos enfoques alternativos han contribuido a prolundizar la discusión — principalmente en la teoría sociológica- de la compleja relación entre agencia y estructura, entre discurso y movimiento. Este arlículo intenta explorar críticamente las construcciones discursivas (ideológicas) acerca de la noción de "desarrollo", las cuales en los últimos años se han constituido en el hase sobre la cual son impulsados los programas contra la reducción de la pobreza en innumerables comunidades rurales del Tereer Mundo.

\section{I}

Como punto de partida, se puede afimar que las melas de este lipo de programas descansan en ciertos presupuestos acerca de lo qué es "desarrollo" y en la lormulación programática de las necesidades y los deseos de una calegoría social especial, los pobres, como los heneficiarios de dichos programas. Desde la perspectiva de estos programas, "desarrollo" comprende una amplia gama de 
metas cualitativas, las cuales contemplan: fortalecer la participación popular, prolundizar la democracia local, mejorar las condiciones de salud y bienestar social y It consolidación del compromiso de la comunidad a desarrólarse. Sus objetivos centrales de "crecimiento con equidad" y "mejorar las condiciones generales de las comunidades rurales" lienen que ser logrados vía la ejecución de intervenciones especílicas a través de una variedad de sectores, utilizando una combinación de esquemas de gestión de arriba hacia abajo y de enfoques parlicipalivos. Estos objelivos, y los supuestos que los delinen, se centran en un concepto de "desarrollo participativo" que se constiluye en medio y en fin de las intervenciones del desarrollo. En la aclualidad, este lipo de intervenciones no se reducen a un programa o a una institución en particular. Por el contrario, el discurso del desarrollo participativo ha venido a constituirse, desde la década pasada. en la nueva ortodoxia de los círculos del desarollo: desde las ONG hasta el Banco Mundial (Lidwards y Hume 1996).

Si hien, como parece obvio, un compromiso de parlicipación simplemente trac consigo el asegurar algún grado de participación de los beneficiarios involucrados en la planilicación e implementción del proyecto, el concepto de desarrollo participativo confrecuencia no iría lan lejos. La mayor parte de la literatura sobre desarrollo participativo, y de la clase de programas que legitima, descansa en una construcción fillosófica muy parlicular acerca de la participación como la iniciativa que lleva al empoderamiento (empowerment) de las comunidades pobres como parlícipes en el proceso de desarrollo. De acuerdo a esta construcción la relación causal entre participación y empoderamiemes, en última instancia depende del establecimiento de una relación espúréa entre conocimiento y agencia, en la cual agentes individuales son "embestidos de poder" a nivel de la conciencia, en un espacio divorciado de la verdadera acción social y polílica. Tales enfoques priorizan la participación con respecto a la producción del conocimiento en lugar de impactar directamente en la gestión de programas $y$, al hacerlo, se conceptualiza a las comunidades seleccionadas para las intervenciones del desarrollo como agentes pasivos que se encuentran esperando la intervención emancipatoria de las organizaciones de desarrollo. A pesar de la alirmación de la relórica del empoderamiento, las comunidades pobres carentes de la capacidad de llevar a cabo la transformación social por ellas mismas, solamente pueden participar en el desarrollo por medio de las agencia que proporcionan las estructuras institucionales que promueven la participación

Con base en la premisa de los supuestos logros oblenidos por el "desarrollo" a través de la participación de las comunidades, principalmente en el contexto de los programas implementados localmente, la nueva ortodoxia del desarrollo contribuye a la perpeluación de los problemas críticos que busca resolver, al no tomar en cuenta las amplias restricciones económicas e institucionales - del capitalismo de la periferia- que aseguran el continuo empobrecimiento de las comunidades rurales de África, Asia y América Latina (Arce et al. 1994). 
Desde mediados de los ochenta, la noción de "participación" se ha convertido virtualmente en sinónimo de "desarrollo" en el discurso de las organizaciones (jue promueven el desarrollo y en el de sus porlavoces y especialistas. Incluso, organismos bilaterales y multilaterales de inspiración conservadora rutinariamente invocan el concepto como un requerimiento básico que debe incorporarse en los escuemas de los proyectos y en los procedimientos de evaluación ( Narayan y Srinivasan 1994). Ahora bien, lo que "participación" realmente implica diliere en gran medida entre dilerentes agencias e instituciones (Rahnema 1992). Para algunos, "participación" simplemente incluye un involucramiento de los heneliciarios en el proceso de planilicación e implementación, a menudo a través de procedimientos de consulta selectivos y limitados. Para otros, alineados con lo que Booth ha denominado el "punto de vista de la ONG" (1994: 24), participación se ha convertido en un imperativo moral, el cual, como precondición para el empoderamiento, lacilita el mismo desarrollo (Edwards 1994: 282). Esta noción de empoderamiento, parece, a primera vista, relativamente no problemática. Los pobres, alejados de los centros de lomas de decisiones dominados por las elites con sus intereses particulares, deben ser empoderados para participar en las decisiones que los afectan. Esto puede lograrse por medio de la participación en las estructuras de control en las organizaciones locales y de las instituciones políticas a todos los niveles. Sin embargo, el empoderamiento, desde esta perspecliva, no necesariamente supone la participación en movimientos polílicos que persigan cambios radicales en la estructura social, ni tampoco en una militancia activa en organizaciones políticas progresistas'. En el mismo sentido, esto puede ocurrir también en un contexto local, entre "los pobres", a través de la formación de su conciencia crílica vía lécnicas parlicipativas de generación y reflexión de conocimiento. El "desarrollo" no es, desde luego, un simple proceso de cambio directo que lleva a cierla clase de transformaciones económicas y sociales, sino que depende del logro de una serie de translormaciones morales en la conciencia de la gente que participa, como agente de cambio en el proceso de desarrolloº . Consecuentemente, la tarea correcta de las organizaciones del desarrollo y de su personal es facilitar las Iransformaciones necesarias en la conciencia, lo cual implica empoderar a los pobres como actores sociales de manera que puedan gestionar y lograr el cambio en sus condiciones de vida.

Las metodologías participativas son actualmente utilizadas en una cantidad diversa de proyectos y programas por ONGs, agencias internacionales y organizaciones multilaterales. Los resultados han sido mixtos, pero generalmente apoyan la noción que la participación con relación a las estructuras de involucramiento de los heneficiarios puede contribuir la efectividad de los proyectos. Los programas participativos parecen ofrecer un lugar para los bencficiarios o sus representantes en algunos aspectos del diseño y de la ejecución de un proyecto, y pueden reinvidicar un compromiso con los intereses de los involucrados. La

Desanollo porticipattvo y la apropioción de la agencia: Uno exploroción sociológlco 
participación tiene otras ventajas para la gestrón de un proyecto. Incluso, un involucramiento mínimo de la comunidad puede reducir costos para los donanles. contrihuyendo a la electividad y recuperación de los costos "compartidos", y a una sostenibilidad de largo plazo (Oakley el al. 1991). Los impactos sobre el ('mipodaremiento popular es menos claro (Rahnema 1992), y, dado su peso moral. Iodavía más dilícil de evaluar. Más importante aun, los supuestos que delinen el concepto de desarrollo participativo en la tradición del imperativo moral, cuya hase de sustentación es la causalidad implicila entre participación y empoderamiento, liene lodavía que ser críticamente examinada (Richards 1995: 16). I:sto es en gran medida porque la participación es moralmente atractiva y polílicamente aceptable para los luncionarios del desarrollo y para los intelecluales que desean un mundo mejor.

Además, una versión crítical del concepto, y los supuestos en los que descansal. parecería generar unas interrogantes incómodas acerca de la existencia del desarrollo como una prolesión y como un proceso de intervenciones técnicosociales. Is decir. o los pohres son capaces ellos mismos de transtormar su propia conciencia y de llevar adelante su proceso de empoderamiento para lograr el desarrollo. como la ideología de la participación lo asume, o, por el contrario no lo son. y deben depender de las intervenciones de "agentes de cambio” prolesionales como lacilitadores externos de este proceso (Goulet 1989). lista paradoja en el corazón mismo de la relórica de la participación, da cuenta de una negación acerca de la capacidad de los pobres de ser la agencia con la capacidad de realizar un cambio social por ellos mismos y en sus propios términos (Ralhnema lo9):122). Como el “desarrollo genuino" lo señala solamente en cicre lipo de transtormaciones, generalmente aquellos lipos mediados por las organizaciones que trabajan dentro de la tradición del imperativo moral, la agencia puede ser loggrada a larés de estructuras importadas de participación.

\section{III}

l: hecho que los "pobres" dehan, en ćltima instancia, depender de agentes cxlcrnos en su lucha por el empoderamieme, descansal en una particular construcción lautológicia de la relación entre conocimiento y agencia, la cual separada del estudio empírico y de la teoría social. ha evolucionado en gran medida dentro de los conlines del dominio del desalrollo allamente sospechosal de la discusión académical y de lo que se consideran investigaciones irrelevantes (Bchbinglon 1994:202). (onsiderando que los modelos de desalrrollo derivados de los paradigmas de la economía neoclásica alribuyen la agencia al individuo como acer racional. por ejemplo maximizando las actiludes empresariales de los productores rurales. el modelo de desarrollo desde el enloque del imperativo moral alribuye la agencia a comunidades de personas pobres que, se asume nostílgicamente, viven encerrados en cadenas de parentesco totalizantes y en la solidaridad mecianica de las "Iradicionales" sociedades campesinas. A pesar de

\section{0}

Realidad 84, 2001 
tener posiciones políticas divergentes, los dos enloques comparten una comprensión de la agencia notablemente similar, comprensión de la agencia (Booth 1994:13). Los agentes activos son agentes con conocimiento, empoderados por medio de su racionalidad para convertirse en aclores sociales efeclivos (Arce el al., 1994:156). Como agencia colectiva es crucial para el desarrollo comunitario; en tal sentido, las intervenciones participativas están principalmente preocupadas por la construcción de un concimiento correcto como una precondición para su logro. Desde la perspectiva del desarrollo participalivo, en vista que "los pobres" heneficiarios direcos del proyecto ocupan una área geográlica especílica en donde el proyecto se lleva a cabo, se asume que su conocimiento es "local". Paradójicamente, los "pobres" necesilan agentes de cambio para lograr el desarrollo ya que se ven obligados a esta asistencia debido al lipo "especial" de conocimiento que contiene las semillas de su empoderamiento.

Inicialmente concebida como un correclivo a las intervenciones técnicas de los expertos en gestión que privilegian los discursos cientílicos de occidente, marginando de esta manera las experiencias de los procesos locales, el concepto de conocimiento local está intrinsicamente relacionado con la historia de los enfoques participativos del desarrollo. Los primeros investigadores y publicistas se plantearon demostrar que, no solamente los pobres eran conocedores, sino que sus estrategias de producción agricola era con liecuencia óplimas para su ambiente ecológico y económico. I a expansión de los enloques "I os Agricultores Primero y la Agrícultura Indígena/Conocimiento Técnico" (conocidos por su siglas en inglés IAK/ITK) durante los años ochental. estableció una ruta en los replanteamientos sobre el desequilibrio existente en las estralcgias de desarrollo. Desalortunadamente, hubo una debilidad. I a húsqueda de sistemas resultó en el entrentamiento de la intormación con conocimiento y la competencia lécnical con molivaciones sociales (Richards 1993:(2). Al igual que el paradigma del agricultor ignorante que estos enloques condenaron. la liaclibilidad del IAK como lécnica analílica dependió de una representación similar, en esta ociasión de su "alter ego"” el agricultor conocedor.dispuesto a experimentar, pero cuyas prácticals estaban lirmemente enraizadats en su "Iradicional", y por lo lamlo sólida, cultura local y en su conocimiento ecológico (Fairhead 1993: 187).

Li conceplo de conocimiento local lue gradualmente expandiéndose hasta intentar abarcar otra clase de conocimientos, lo cual, se pensó, podría lormar la base de un conocimiento capar de generar el empoderamiento comunitario y la transformación social. "El conocimiento local que es único paral una cultura o sociedad particular" era el asidero para conslituir un "recurso crílico para el desarrollo. porque éste "constituye la información de hase de una sociedad. lo cual facilita la comunicación y la loma de decisiones" (Warren et al. 1995: xiv). De la misma manera que el conocimiento agrícola local podría extracrse a través de lécnicas especializadas como la Valoración Rural Rípida (VRR), el conocimiento local en general podría ser oblenido a lavés de las metodologías 
participativas, formando la hase para el diseño y cjecución de proyectos, lo cual se sostuvo, podría ser apropiado localmente, así como también ser sostenible (Chambers 1983). Durante los últimos quince años un complejo híbrido de enfoques, derivados de las lécnicas asociadas con la VRR de los agrónomos del desarrollo por un lado, y la Investigación Acción de los aclivistas influenciados por las ideas de Paulo Freire por el otro, ha evolucionado, inicialmente a la Valoración de la Participación Rural (VPR) y recientemente en el Aprendizaje Participativo y Acción (APA).

La expansión rápida del desarrollo participativo ha resultado en el establecimiento de una gama de práclicas autorizadas para su implmentación junto con un grupo de lécnicos prolesionales que las promocionan (Richards 1995). Asumiendo con autoridad la premisa que los "pobres" ya saben cuáles son sus problemas y que además lienen un conocimiento mejor de su realidad que los "expertos" de lucra, las lécnicas participativas procuran tener acceso y consolidar un dominio del conocimiento "local" como lundamento de un tipo de rellexión crílica que pueda llevar a la acción social. La investigación ya no es concebida como el proceso que intenta acortar la distancia entre sujeto y objeto, sino como un proceso de aprendizaje e interacción mutuo (Nelson y Wright 1995: 58). La evaluación participativa ha sido suplantada por el aprendizaje participativo y por la investigación vinculada a proyectos, la cual es llevada a cabo por lacilitadores con la colaboración de los mismos "pobres". Las técnicas del APA localizan poblados o grupo de personas en la misma área administrativa, así de esa manera se los constituyen en comunidades, y trabajan para construir una representación de sus problemas inmedialos, de la economía y de la organización social. Cada una de las técnicas empleadas (por ejemplo, calendarios estacionales y clasilicación de riquezas) asumen que las obligaciones sociales y los sistemas agrícolas operan en un área restringida, que las comunidades son orientadas hacia lo local, y éstas no se dan cuenta que la diversidad en las estrategias de las lormas de vida de las gentes no son una simple reflexión de una dilerenciación en la riqueza (Mosse el al., 1995). Estas técnicas se centran en el papel de los lacilitadores, en la construcción de dominios restringidos de un conocimiento local que sea relevante para la implementación de los proyectos y en la perpeluación de estrategias de desarrollo rural centradas en proyectos y en poblaciones previamente localizadas.

Si bien la perspectiva política radical de la investigación-acción inspirada en los trabajos de Freire pudo haber proporcionado la legitimación inicial a las melodologías participalivas, la transición "desigual"de la VRR a la VPR signilica que, en la práclica, el desarrollo participativo liende a despolitizarse, y se convierta en otra intervención técnica de la ingeniería social. Además, no existe evidencia concrela que indique con eertera que las metodologías participativas llevan realmente al empoderamiento. Como Richards lo señala, en relación a la VPR. no solamente sus supuestos teóricos son inverosímiles y sin una base

\section{2}

Realidad 84, 2001 
sólida, sino que "sus afirmaciones desacansan más en la fé que en la ciencia" (1995: 15-16). Al igual que el mismo desarrollo comunitario cuando es confinado a un contexto de proyectos localizados, el empoderamiento a través de la participación es una fantasía divorciada de la acción polílica del cambio; en tal sentido, la comunidad local es imaginada —románticamente- como una masa homogénea de gente pobre con una tendencia inherente a la acción colectiva (Eyben y Lanclhury 1995: 194).

Incluso cuando es liherado del peso del empoderamiento, el concepto que sobre el conocimiento liene la ideología del (lesarrollo local, percibielo como un lipo distinto de conocimiento y que es potencialmente acessible via técnicas especializaldas, se mantiene problemático. Denota una versión rígida y sistemalizada del conocimiento, abstraido de los agentes individuales. lo cual le impide reconocer las (leterminaciones históricas de su evolución (Agrawal 1995: 421), y lija exclusivamente su alención en el presente del proyecto. Al centrarse en lo "Iocal" como una entidad limilada en tiempo y espacio, el "conocimiento" de la gente "local"se presume que no es únicamente derivado de su ambiente, sino que es relevante para una localidad especillica. Las técnicas del desarrollo que intentan acceder al conocimiento "local" enlatizan y crean límiles rígiclos entre lo qué es "local" y relevante para el proyecto, y lo qué se asume que no es local y por lo tanto irrelevante para el proyecto. Un concepto más generalizaldo de conocimiento local, derivado de la antropología, es igualmente estrecho. Fn este caso. se construye el conocimiento local como un conocimiento cultural restringiclo, cuyo portador son los residentes de localidades especílicas que comparten una aliliación cultural. Isste conocimiento es a menudo considerado de mayor relevancia (que el conocimiento técnico "occidental", asi como estar más cercano a lo auténtico y "uradicional". Podría alirmarse, descle la perspectiva del conocimiento antropológico, que los proyectos fracisán porque no toman en cuenta el conocimiento locial.

Tanto las construcciones de la ideología del desarrollo como las del conocimiento antropológico sohre el "conocimiento local", descansan en una concepción de las comunidades locales como entes colectivistas y productoras de "conocimiento". Al lormular una oposición entre conocimiento "occidental" y local enfrentan los objelivos básicos del proyecto y las estralegias lécnicals con un lipo especial de conocimiento. En realidad, lo que la gente en cualquier región "conoce", generalmente, incluye una gran cantidad (lel llamado conocimiento cientílico "occidental" y otros conocimientos, así como diversals estrategias locales que están relacionadlas con su ambiente agrícola, cultural. económico y social (Agrawal 1995). Estos dos lipos de conocimiento no son separables ni discretos, sino que lorman parte de los recursos del conocimiento total de una

Desarrollo participativo y la apropiación de la agencia: Una exploración sociológica 
persona en un tiempo daclo. Asimismo, cierta clase de conocimientos puede ser articulado solo en contextos sociales especílicos.

İn mínthiples comunidacles rurales, la persona "local"que vive en un área la mayor parte de su vida es probable gue tenga nexos sociales que se extiendan a tratés de locialidades y regiones, justamente de la misma manera que él o ella prohahlemente haya desarrollado estrategias de ingreso que van más allá de su localidald. Lin contraste a las representaciones de los "sistemas" de conocimiento local creados por medio de las metodologgías participativas, lo que la gente de una localidad conoce ni es especílicamente locial ni es unilormemente manteni(o. De igual manera. las limilaciones gue la gente enlienta para lograr mejores condiciones de vida no se circunscriben a las localidades particulares o la inlerrención de los proyectos. Issto liene serias implicaleiones para la electividad de las estrategials de (lesarrollo (jue persiguen utilizar las nociones de empoderamiento comunitario a través de el conocimiento locial como la base y justificación de intervenciones localizadlas. Dalda la "capacidad de un contexto deslavorable para (lisipar los benclicios de los esluerzos locales" (Booth 1994: 6), las lécnicas que lienen como objetivo aceeler al conocimiento "local" como la clave para los prohlemas y soluciones del desarrollo, en contextos lociales, pueden prescindir del punto en su totalidatd. In la actualidad es ampliamente aceptato que las macro-polílicals y las variahles polílicals ejerran "una inlluencia crílica en los procesos de desartollo, contabilizando los diferentes resultados en escenarios sociocconcinicos similares" (Booth 1994: 8). I a construcción del conocimiento locial y la participación local como estrategias claves para empoderar a las comunidades locales, establece como prioridad la "ignorancia polítical, uhiciando los problemas del desirrollo y sus soluciones. Estos se convierten en convenientemente suceptibles a la clase de intervenciones en proyectos locales que son lícilmente controlables y políticamente convenientes.

la ignorancia política lambién hace posible la construcción de imágenes de comuniclades que pueden, y podrán, actuar colectivamente. Esta clase de alirmaciones derivalas de una interprelación equivocada de los aspectos hásicos de la teoría sociológica y de una antropología pasada de mola, no lienen ningún lundamento. Iśn dilerentes partes del mundo perilérico, mientras la acción coleceliva es una posibilidald, ésta - comunmente- se lleva a cabo en un contexto donde participan dilerentes comunidades a través de organizaciones que trascienden límites geogrílicos locales. La expresión concrela de estas organizaciones son, por ejemplo, cooperalivas, movimientos polílicos, y organizaciones religiosals. I a prioridad de lo local y micro sobre lo meso y lo macro electivamente oscurese y considera irrelevante el contexto en el cual la gente de las áreas rurales se encuentra, un contexto en gran medida determinado por la articulación (le macro-políticas y niveles meso-institucionales en los que las comunidades rurales son actores (Bebhington 1994, Booth 1994). El contexto micro de los estudios del (lesarrollo y cierı lipo de prácticas antropológicas lo definen en

\section{4}

Realidad 84, 2001 
realidad como una calegería analílica. No es una descripción de la realidad social. I a interacción de los dilerentes niveles institucionales no es ni de atriba hacia abajo ni de abajo hacia arriba, sino cue puede ir en cualquier dirección. dependiendo de las circunstancias. Que éstos con liecuencia sean amontonados contra el peldaño más hạjo, no es debido a una inherente calusalidad de lo macro sobre lo micro, sino de los intereses y lealtades de agentes sociales especílicos que operan a niveles intermedios. I a comprensión de la verdadera dinámica de la interacción entre niveles requiere considerar más que una simple noción de vinculación entre lo micro y lo macro, lo cual continúa privilegiando lo locial y lo micro. Al mismo liempo que se insinua unal rígida separación de lo cyue de hecho son aspectos de una realidad compleja y multilacélica (Boolh 1994: 18).

In una siluación donde la operallividad de las barterats instilucionales y económicas exlra-locales deline que la agencia individual es insuliciente para lograr el "desarrollo" (Behbinglon 1994: 21()-12). las intervenciones en proyectos y el aceso a la asistencia malerial de la cual es parte se convierten en los medios crílicos para superar los obstículos extra-locales. Así, el camino correcto para lograr el desarrollo — de la manera en que es definido por los actores localescontribuye a reproducir el milo (que justilical las intervenciones como el medio más idóneo para solventar los problemas e impactando la apropiación de la agencia por las organizaciones del desarrollo. Las aspiraciones para lograr el desarrollo personal no son ni obslaculizadas ni lacilitadas por el conocimiento "Iocall". I a capacidad de una persona de ser agencia depende de reglas y recursos económicos y políticos y no solamente de su aceeso al conocimiento (Bchbington 1994: 21()-12). En cualquicr calso, el conocimiento no es unal caltegoría abstracta de recursos que automálicamente empodera a la persona que liene aceeso al mismo. La utilidad del conocimiento es limitado por las actividatdes que llevan a cabo las instituciones sociales y que hacen accesible o inutilizathle cierta clase de informaciones.

En la mayoría de las comunidades lociales de la perileria, la gente se encuenlaa en condiciones deslavorables por la lialta de información con respecto a los precios, oportunidades de mercado, mareo legal y a sus derechos como ciudadanos, estos últimos que le garantizan su participación en las instituciones políticals. La incertidumbre acercia de los derechos de representación, el poder relaltivo de los representantes electos y la falta de una representación efectiva conspiran para mantener a la gente encadenada a un sistema que los explota, creando electivamente las condiciones hajo las cuales lo macro y lo meso determinan el universo de lo micro. La situación por la que atraviesan las comunidades rurales pobres se ha tornado más complicada por la institucionalidad neoliberal; es decir, por la implementación de polílicas de ajuste macroeconómico que han contribuido a un crecimiento desequilibrado y que han generado políticas contradic-

Desarrolloparticipativo y la apropiación de la agencia: Uno exploroción sociológica 
torias a nivel local y regional. No es ninguna sorpresa, entonces, que los agentes rurales le presien poca alención a la participación comunitaria y que continúen (lándole ptioridad a la asistencia material generada por las intervenciones asociadas con los proyectos, constituyendose ésta como un medio para lograr lo que los agentes externos definen como el resultado ideal de los objelivos perseguidos.

A pesal (lel hecho que las intervenciones fracasan al no integrar en sus escenarios el amplio contexto (del subdesarrollo, las mismas proporcionan una oportuniclad para cjue una minoría pueda alcanzar el desarrollo personal en sus propios lérminos. El resultado es la perpeluación de la dependencia y la consolidación de la desigualdad; en otras palabras, se logra el empoderamiento material de algunos y no el empoderamiento genuino de la gran mayoría. Esta situación no es inevitable. El (lesarrollo participativo y el empoderamiento de las comunidades rurales es una posibilidad, pero su realización depende del reconocimiento que el empoderamiento solo puede ocurrir a través de la participación en las estructuras de loma de (lecisiones en un eontexto polílico amplio. Asumir el empoderamiento al nivel de la conciencia y reproducir la letichización del conocimiento local asigna a las comunidades locales el estatus de clientes y por lo lanto de ser dependientes pasivos de las intervenciones del desarrollo. En la medicla que las prácticas del desarrollo ignoren los ambientes macroeconómicos y las estructuras polílicas que los crean, los pobres en muchas regiones continuaraín dependiendo de la asistencia de los organismos del desarrollo y su proceso de empoderamiento real se verá trunciado.

\section{NoTAS}

1. Como Goulet lo scñala, dicha participación es políticamente amenazante para muchos gobiernos y podría no ser tolerada en un nivel macro, si bien podría ser estimulada en contextos micros (Coulet 1989).

2. Para un tener una visión coherente de cómo este proceso se percibe, ver los argumentos de Friedman, quićn. a pesar de reconocer la importancia del contexto político general para avanzar hacia el "desarrollo" por medio del empoderamiento, asume que el empoderamiento social, local y psicológico lógicamente precede a la consolidación de "la capacidad polílica del campesino" (1992; 33-6).

3. Estas representaciones siempre son construidas desde una posición patriarcal, ya que se asume que los agricultores son hombres y que las mujeres, como Irabajadoras agrícolas, tienen muy poco que aportar en el proceso de toma de decisiones.

4. Como Mosse et al. (1995) lo indican, se suponía que los dos enfoques eran incompatibles debido a que fueron diseñados para diferentes fines; la VRR para una obtención rápida de la información agrícola y la investigación-acción para la concientización.

\section{REFERENCIAS BIBLIOGRÁFICAS}

Agrawal, A. (1995) "Dismantling the Divide between the Indigenous and Scientific Knowledge", Development and Change 26: 413-39. 
Arce, A.. M. Villarreal y P. de Vries (1994) "The Social Construction of Rural Development". en D. Booth (ed). Rethinking Social Development: Theory. Research and Practice, 1.52-71. I larlow: Longman.

Behbing(on. A. (1994) "Theory and Relevance en Indigenous Agriculture: Knowledge. Agency and Organization”, en D. Booth (ed.) Rethinking. Social I)evelopment: Theory: Ressearcl ancl Practice. 2()2-25: Ilarlow: I ongman.

Boolh. D. (1994) "Rethinking Social Development: An Overview", en D. Booth (ed.) Rethinking Social Deselopment: The'orv, Resecarch and Practice, 3-34. Harlow: Longman.

Chambers. R. (1983) Rural I)evelopment: Putting the Last First. Londres: Longman.

Edwards. M. (1994) "Rethinking Social Development: The Scarch for 'Relevance'”. en D. Booth (ed.) Rethinking Social Inevelopment: Theorv, Resecarch and Practice', 27997. Harlow: Longman.

Edwards, M. y D. Hume. (eds) (1996) Beyond the Magic Buller" NGo) Performance and Acrountalilits in Post-Cold World. West Hartford,C.T: Kumarian.

Eyhen, R. y S. Ladlhury (1995) "Popular Participation in Aid-Assisted Projects: Why More in Theory and Practice"", en N. Nelson y S. Wright (eds.) Pow'er and Participatory I) Ie'elopment, 192-20)(). Londres: Intermediate Technology Publications.

Fairhead, J. (1993) "Representing Knowledge: The "New Farmer" in Research Fashions". en J. Pollier (ed.) Practising Development: Social Science Perspectives, 186-204. Londres: Routledge.

Ficdman. J. (1992) Eimpowerment: The Politic:s of Alternative Development. Oxford: Clarendon.

Goulet, D. (1989) "Participation in Development: New Avenues". World Deverelopmenem 17 (2): $16.5-78$.

Long. N. y A. Long (eds). (1992) Batrlefüelds of Knowleclge: The Interlocking of Theory and practice in Social Research and Development. Londres: Roulledge.

Mosse. D. (1994) "Authority, Gender and Knowledge: Theoretical Reflections on the Practice of Participalory Rural Appraisal". Development and Change 25: 497-526.

Narayan,D. y L. Srinivalsan (1904) Participatory Development Tool Kit: Materials to Facilitale Community Empowerene'm, Washington, DC: World Bank.

Nelson. N. y S.Wright (1995) "Participatory Research and Participatory Observation: Two Incompalible Approaches", en N. Nelson y S. Wright (eds) Power and Participatory I Development, 4:3-59. Londres: Intermediate Technology Publications.

Oaklcy, P. et.al. (1991) Projects with People: The Practice of Participation in Rural Development. Ginchra: International Labour Olfice.

Rahnema, M. (1992) "Participation", en W. Sachs (ed.) the Development Dictionary: A Guide to Knowledge as Power, 116-31. Londres: Zed .

Richards, P. (1993) “Cultivation: Knowledge or Performance?”, en M.Hobart (ed.) An Anthropological Critique of development: The Growth of Ignorance, 60-78. Londres: Routledge. 24 (Octubre): 13-16.

Warren, D., L. Slikerveer y D. Brokensha (1995) "Introduclion", en D. Warren, L. Slikveer y D. Brokensha (eds.) The Cultural Dimension of Development: Indigenous Knowledge Systems. Londres: Intermediate Technology Publications.

Desonollo porticipórivo y lo oproploclón de lo agencia: Uno exploroclón soclológico 